\title{
ÍNDICE DE DESEMPENHO COMPETITIVO DAS EMPRESAS DE MÓVEIS DA REGIÃO METROPOLITANA DE BELÉM ${ }^{12}$
}

\author{
Zilda Joaquina Cohen Gama ${ }^{3}$ \\ Antônio Cordeiro de Santana ${ }^{4}$ \\ Fernando Antônio Teixeira Mendes ${ }^{5}$ \\ Ahmad Saeed Khan ${ }^{6}$
}

\begin{abstract}
Resumo - A indústria de móveis da Região Metropolitana de Belém é intensiva em emprego e ajuda a reduzir os impactos ambientais das serrarias, uma vez que utiliza as aparas e resíduos de madeira dessas empresas como matéria-prima. A análise fatorial foi utilizada para gerar um Índice de Desempenho Competitivo e determinar a posição competitiva dessas empresas na indústria de móveis. Os resultados mostraram que a maioria das empresas da indústria de móveis encontra-se em posição intermediária de desempenho competitivo, em razão das limitações de fatores, como falta de mão-deobra qualificada, baixo nível tecnológico, dificuldade de acesso ao crédito e baixo grau de integração vertical e horizontal da cadeia de suprimento. Finalmente, conclui-se que apenas uma empresa apresentou alto grau de desempenho competitivo.
\end{abstract}

Palavras-chave: Índice de Desempenho Competitivo (IDC), análise fatorial, empresas de móveis, tecnologia, Estado do Pará.

Recebido em 20/10/2006. Aceito em 14/02/2007.

2 Os autores agradecem às sugestões de dois revisores anônimos da Revista. Os possíveis erros remanescentes são de responsabilidade dos autores. Artigo elaborado a partir da dissertação de mestrado em Economia do primeiro autor, pela Unama.

3 M.Sc em Economia pela Universidade da Amazônia - UNAMA - E-mail: zildagama@ yahoo.com.br.

4 Professor da Universidade Federal Rural da Amazônia - UFRA - E-mail: santana@ nautilus.com.br.

5 Professor da Universidade da Amazônia - UNAMA - E-mail: fernando@ufpa.br.

6 Professor da Universidade Federal do Ceará - UFCE. 


\section{Introdução}

A indústria de móveis brasileira é formada por 16.104 micro, pequenas e médias empresas formais (17,9\% do total), envolvendo 206.352 empregos e um faturamento de US\$ 5,61 bilhões em 2005. Neste mesmo ano, exportou US\$1,003 bilhão (Abimóvel, 2006).

Nesse cenário, a indústria de móveis do Estado do Pará, formada por 106 micro e pequenas empresas formais $(32,7 \%$ do total), gerou 721 empregos, exportou US\$ 7,60 milhões em 2005 e ocupa a sétima posição no ranking dos Estados exportadores.

O Estado do Pará, historicamente, figurou como grande produtor e exportador de madeira para outros Estados e para o mercado internacional. Entretanto, para uma média de 700 mil toneladas/ano exportadas de madeira serrada e/ou semi-elaborada, a indústria do Pará processa apenas $0,6 \%$, que destina à produção de móveis, artefatos e utilidades de cozinha com maior valor agregado (Santana, 2002; Sebrae, 2004; Gama, 2006).

Atualmente, o Estado do Pará conta com dois pólos moveleiros: um situado no município de Paragominas; e o outro, objeto deste trabalho, localizado na Região Metropolitana de Belém (RMB), englobando os municípios de Belém, Ananindeua, Marituba, Benevides e Santa Bárbara. Ambos são frutos do processo recente de reestruturação da indústria madeireira. Segundo Santana (2002, 2003), algumas das empresas de madeira serrada e beneficiada estão fabricando móveis e artefatos na mesma fábrica ou em unidades separadas da mesma empresa, aproveitando as sobras e resíduos da madeira serrada. Anteriormente, esse resíduo era queimado, com uma parcela destinada à lenha e outra transformada em carvão. Com o objetivo de atenuar esses problemas ambientais causados pelas madeireiras, o Sebrae e o Governo do Estado iniciaram a organização do tecido de micro e pequenas empresas em cluster, fazendo a articulação das moveleiras às serrarias certificadas, que se comprometeram a fornecer madeira de qualidade e oriundas de áreas sob manejo florestal 
Zilda Joaquina Cohen Gama, Antônio Cordeiro de Santana,

Fernando Antônio Teixeira Mendes \& Ahmad Saeed Khan

sustentado (Carvalho; Santana, 2004). Essa tentativa de reorganizar a indústria moveleira no Estado do Pará iniciou-se neste século e não se dispõe de parâmetros indicando o grau de competitividade dessas empresas.

As micro e pequenas empresas da RMB analisadas por Carvalho e Santana (2004) não fazem o desdobramento da madeira em tora, portanto não se vinculam diretamente aos problemas ambientais provocados pela extração de madeira da floresta amazônica. A matéria-prima que utiliza é adquirida no "lixão das serrarias", ou seja, elas adquirem as aparas e sobras de madeira das serrarias, bem como compram caçambas de tábuas, pranchas, barrotes, etc., envolvendo todos os tipos de madeira. Nesse aspecto, as movelarias estão exercendo uma ação mitigadora dos efeitos ambientais das serrarias, pois em outros tempos esse resíduo era simplesmente queimado, transformado em carvão ou lenha. A disponibilidade de madeira para as movelarias foi um dos principais entraves ao crescimento dessas empresas revelado na pesquisa ${ }^{7}$. Por que isso ocorre? As movelarias demandam pouco volume de madeira e as serrarias não estão interessadas em atender essa demanda. Quando vendem, em casos especiais, não dão nota fiscal. Em razão disso, as moveleiras estão utilizando somente o indispensável de madeira densa na fabricação de móveis e importam placas e painéis de madeira das regiões Sudeste e Sul. Há casos em que a matéria-prima importada representa mais de $60 \%$ do custo de produção. Com relação ao resíduo produzido pelas moveleiras da RMB, boa parte está sendo destinada para cama de galinheiro, outra parte é transformada em briquete para produzir energia, outra parte é utilizada como lenha para aquecer a estufa de secagem de madeira e o restante é recolhido para o lixão pela prefeitura.

\footnotetext{
As madeireiras da Região Metropolitana de Belém, sobretudo as situadas nos distritos industriais de Icoaraci e Ananindeua, têm planos de manejo florestal e algumas estão em fase de certificação. As micro e pequenas moveleiras entrevistadas não possuem máquinas para serrar madeira em tora, portanto não estão vinculadas diretamente à exploração florestal. Até o momento, a bitola de seis metros adotada pelas madeireiras não atende às moveleiras que necessitam de bitolas menores. Esta bitola acarreta grande desperdício no momento da extração, uma vez que parte do fuste da árvore e os galhos não são aproveitados. A quantidade média de madeira densa serrada utilizada pelas micro e pequenas moveleiras da RMB é inferior a $22 \mathrm{~m}^{3}$ por ano, envolvendo cerca de 10 espécies de madeira. Este volume de madeira é mais de 200 vezes inferior ao de uma serraria de pequeno porte, conforme Santana (2002).
} 
Boa parte das empresas trabalha por encomenda e foca o preço e a qualidade do produto como elementos fundamentais da estratégia de concorrência. A dificuldade de acesso à matéria-prima, a baixa integração cooperativa e a incipiente conexão com os elos da cadeia a montante e a jusante tornaram as moveleiras que fabricam móveis de madeira densa ameaçadas pelos móveis tubulares (móveis de ferro) e de plástico, para o mercado local. Em razão disso, iniciou-se um processo de organização dessas empresas para uma integração vertical e horizontal de alguns elos da cadeia produtiva.

$\mathrm{Na} \mathrm{RMB}$, as moveleiras operam com móveis retilíneos, de estilo colonial e rústico. $\mathrm{O}$ design moderno também está presente, assim como o modulado. Esse fato implica que as empresas estão operando em níveis de competitividade diferentes.

O objetivo deste trabalho foi determinar a posição competitiva das empresas de móveis da RMB, empregando o índice de desempenho competitivo (IDC) utilizado por Santana (2006) para analisar o desempenho competitivo das empresas da indústria de polpa de frutas paraense. A metodologia utilizada para alcançar esse objetivo foi a análise fatorial. Os dados foram obtidos em pesquisa de campo, realizada em uma amostra representativa de 53 empresas no ano de 2003, por Carvalho e Santana (2004). Do universo amostrado, 45 são microempresas e oito são pequenas empresas, pois não existia nenhuma média ou grande empresa, segundo o número de pessoas empregadas.

O trabalho está organizado em três seções além desta introdução. A primeira apresenta o referencial teórico sobre a competitividade e o modelo de análise fatorial, utilizado na determinação do IDC. Na segunda, discutem-se os resultados obtidos em concomitância com o referencial teórico e, por fim, encerra-se o trabalho com as conclusões. 
Zilda Joaquina Cohen Gama, Antônio Cordeiro de Santana,

Fernando Antônio Teixeira Mendes \& Ahmad Saeed Khan

\section{Metodologia}

\subsection{Fundamentação teórica}

Na literatura especializada em organização industrial existe um conjunto de elementos metodológicos que discute o conceito de competitividade, com base no índice de concentração e poder de mercado, economias de escala e de escopo, estratégias empresariais e confluência de forças sistêmicas que determinam o desempenho competitivo das empresas (Bain, 1968; Porter, 1993; Coutinho; Ferraz, 1994; Esser et al., 1999; Shy, 1997; Tirole 2001; Kupfer, 2004).

Por competitividade empresarial entende-se o esforço realizado pelas empresas para continuarem no mercado, mantendo e/ou ampliando sua parcela de mercado. Esse conceito de competitividade, definido com base nos postulados do modelo estrutura-conduta-desempenho (ECD) ou modelo tradicional da competitividade industrial proposto por Bain (1968), segundo Possas (1987), OCDE (1992), Coutinho e Ferraz (1994) e Farina e Zilbersztaja (1998), não é operacional. Na tentativa de superar essa dificuldade, Porter (1993) considerou que as variáveis que definem as dimensões do modelo ECD atuam de forma dinâmica e não estática, como proposto. Na mesma direção, a OCDE (1992), Coutinho e Ferraz (1994), Possas (1996) e Esser et al. (1999) propuseram que a competitividade empresarial é o resultado da interação sistêmica de conjuntos de fatores que podem ser agrupados nos níveis micro, macro, meso e meta, levando em conta os pontos relevantes das abordagens teóricas tradicionais e modernas (Santana, 2002, 2003).

Sinteticamente, o modelo ECD ou modelo tradicional postula que o desempenho competitivo da indústria é o resultado das relações de uma constelação de elementos organizados nas dimensões Estrutura (concentração de fornecedores e clientes, diferenciação e diversificação de produtos, condição de entrada, formas de integração), Conduta (preços, estratégia de venda, pesquisa, desenvolvimento e inovação, tática geral) e Desempenho (alocação eficiente de recursos, margem de lucro, 
conservação de recursos, bem-estar social, emprego, parcela de mercado). Segundo este modelo, a estrutura determina a conduta e esta, isolada ou em conjunto com a estrutura, determina a posição competitiva das empresas. Entretanto, os elementos do bom desempenho nem sempre são totalmente compatíveis entre si ou se relacionam completamente entre as dimensões, o que dificulta a criação de um índice preciso que possa ser adotado para avaliar as características de desempenho das empresas. Em busca de alternativa, Braga (1980) empregou métodos econométricos para determinar a estrutura de mercado e o desempenho da indústria brasileira, utilizando como variável dependente alguns dos indicadores de desempenho e como variáveis independentes um conjunto de variáveis indicadoras das dimensões estrutura e conduta. Apesar do rigor científico dos indicadores criados, sua aplicação esbarra nas limitações de estatísticas e na exigência de alto conhecimento para a estruturação dos modelos econométricos.

Porter (1993) propôs o Modelo de Cinco Forças (MCF) competitivas, mediante a reorganização dos elementos que influenciam a competitividade empresarial em cinco dimensões ou forças: poder do fornecedor (número de fornecedores, exigência de integração vertical, escassez de fornecimento), poder do cliente (número de clientes, concentração dos compradores, capacidade do setor, homogeneidade do setor), produtos substitutos (marca versus genérico, defasagem de valor-preço para outros produtos), entrada de empresas potenciais (economia de escala, custo de capital elevado, falta de acesso a canais de distribuição, custo de transação elevado) e rivalidade empresarial (concentração do setor, taxa de crescimento, rigidez dos custos, tática de concorrência de preços). Porter entende que as vantagens competitivas devem ser construídas como um fenômeno sistêmico, determinado pela confluência da interação das empresas com seus fornecedores e clientes, visando atenuar as ameaças de empresas rivais, concorrentes potenciais e de produtos substitutos no mercado. Apesar do maior grau de operacionalidade deste modelo, dada a facilidade de obtenção de informações no âmbito operacional da empresa, não se tem um indicador que sumarize o resultado de tais efeitos. O padrão de análise disseminado por Porter 
Zilda Joaquina Cohen Gama, Antônio Cordeiro de Santana,

Fernando Antônio Teixeira Mendes \& Ahmad Saeed Khan

(1993, 1999) e empregado em diversos estudos (Fairbanks; Lindsay, 2002; Silva, 2006) se pauta na análise individual dos elementos de cada força, e o vínculo é tecido mediante análise e alguns cruzamentos entre variáveis.

O modelo de competitividade sistêmica adiciona aos modelos anteriores a influência de elementos como infra-estrutura, política, cultura, inovação tecnológica e de gestão e o ambiente institucional, que atuam em toda a cadeia produtiva, além da dinâmica intertemporal do mercado (Possas, 1987; OCDE, 1992; Coutinho; Ferraz, 1994; Ferraz et al., 1995; Farian; Zilbersztajn,1998; Esser et al., 1999; Santana, 2002). Assim, de acordo com Santana (2001), a abordagem sistêmica da competitividade pode ser considerada como uma paisagem que se move ao sabor da influência dos elementos agrupados nos ambientes de análise microeconômico (eficiência alocativa, capacidade de gestão, estratégias, inovação tecnológica, rede de integração), macroeconômico (política monetária, fiscal, cambial, orçamento), institucional (fatores socioculturais, governança, organização econômica, capacidade política) e de política setorial (infra-estrutura, educação, política ambiental e tecnológica, política regional). Pelo que se observa, a construção de um indicador de competitividade sistêmica tornase ainda mais complexa. Por essa razão, os estudos desenvolvidos por Coutinho e Ferraz (1994), Farina e Zilbersztajn (1998), Santana (2002, 2003), Santos (2002), Gomes (2003), e Carvalho e Santana (2005) utilizaram tabelas de freqüência para apoiar as análises de comportamento dos elementos de cada dimensão da competitividade sistêmica. Como no modelo de Porter, as interações entre as forças competitivas e a avaliação de conjunto se restringem, basicamente, ao campo das análises qualitativas e interpretativas. Portanto, há uma lacuna no que se refere à construção de indicadores de desempenho competitivo capazes de refletir a dimensão do conceito de competitividade empresarial.

Neste trabalho, pretende-se adotar um método de maior rigor estatístico e que permite visualizar a confluência das diversas forças que influenciam a competitividade. Na indústria de móveis de madeira da RMB, é a primeira vez que se emprega a técnica da análise fatorial para construir um índice de desempenho competitivo. 
Modelar essas dimensões não é tarefa fácil e constitui o desafio deste trabalho. A iniciativa foi estruturar um modelo estatístico para resumir a massa de informações em um conjunto reduzido de variáveis capaz de mais bem representar o movimento das empresas da indústria de móveis da RMB na luta para assegurar seu espaço no mercado. As vantagens competitivas de curto prazo, geralmente, estão associadas aos diferenciais de custos resultantes das condições de fatores disponíveis em dados territórios (no caso, disponibilidade de matéria-prima, mão-de-obra barata, crédito subsidiado, etc.). Por outro lado, as vantagens competitivas sustentáveis ou de longo prazo se vinculam a tamanho adequado da planta empresarial, treinamento contínuo de mão-de-obra, qualidade, diversificação e diferenciação da produção, integração vertical e horizontal, visando agregar valor a toda a cadeia produtiva.

Acredita-se que as empresas da indústria de móveis da RMB estão operando com grau de competitividade heterogêneo. A constatação científica da posição competitiva de cada empresa na indústria, porém, não é possível de ser identificada por meio da análise qualitativa por categoria de empresas, segundo o tamanho.

\subsection{Modelo analítico}

A análise fatorial, de modo geral, é utilizada para avaliar a estrutura das inter-relações (correlações) entre um grande número de variáveis, definindo um conjunto de dimensões latentes comuns que facilitam a compreensão da estrutura da nuvem de dados, chamadas de fatores. Com o emprego dessa técnica, inicialmente podem-se identificar as dimensões isoladas da estrutura dos dados e então determinar o grau em que cada variável é explicada por cada dimensão ou fator. Depois dessa etapa, a análise fatorial pode ser empregada para reduzir a massa de dados.

Um modelo de análise fatorial pode ser apresentado na forma matricial, como em Dillon e Goldstein (1984): 
em que:

$\boldsymbol{X}=\mathrm{p}$-dimensional vetor transposto das variáveis observáveis, denotado por $\boldsymbol{X}=\left(\boldsymbol{x}_{\boldsymbol{1}}, \boldsymbol{x}_{2}, \ldots, \boldsymbol{x}_{p}\right)^{\prime} ; \boldsymbol{F}=\mathrm{q}$-dimensional vetor transposto de variáveis não-observáveis ou variáveis latentes chamadas de fatores comuns, denotado por $\boldsymbol{F}=\left(\boldsymbol{f}_{\boldsymbol{1}}, \boldsymbol{f}_{2}, \ldots, \boldsymbol{f}_{q}\right)$ ', sendo q $<\mathrm{p} ; \boldsymbol{e}=\mathrm{p}$-dimensional vetor transposto de variáveis aleatórias ou fatores únicos, $\boldsymbol{e}=\left(\boldsymbol{e}_{\boldsymbol{p}}, \boldsymbol{e}_{2}, \ldots, \boldsymbol{e}_{p}\right)$ '; e $\alpha=$ matriz (p, q) de constantes desconhecidas, chamadas de cargas fatoriais.

No modelo de análise fatorial pressupõe-se que os fatores específicos sejam ortogonais entre si e com todos os fatores comuns. Normalmente, $E(\varepsilon)=E(F)=0$ e $\operatorname{Cov}(\varepsilon, F)=0$.

A estrutura inicial utilizada para determinar a matriz de cargas fatoriais, em geral, pode não fornecer um padrão significativo de cargas das variáveis, por isso não é definitiva. A confirmação ou não dessa estrutura inicial pode ser feita por meio de vários métodos de rotação dos fatores (Dillon; Goldstein, 1984; Johnson; Wichern, 1988). Nesta pesquisa, utilizouse o método varimax de rotação ortogonal dos fatores. O método varimax é um processo em que os eixos de referência dos fatores são rotacionados em torno da origem até que alguma outra posição seja alcançada. $\mathrm{O}$ objetivo é redistribuir a variância dos primeiros fatores para os demais e atingir um padrão fatorial mais simples e teoricamente mais significativo (Reis, 2001; Hair et al., 2005; Santana, 2005).

A escolha dos fatores foi realizada por meio da técnica de raiz latente. Esta técnica parte do princípio de que qualquer fator individual deve explicar a variância de pelo menos uma variável para que seja mantido para interpretação. Cada variável contribui com um valor 1 do autovalor total. Com efeito, apenas os fatores que têm raízes latentes ou autovalores maiores que 1 são considerados significantes, e os demais fatores com autovalores menores do que 1 são considerados insignificantes e descartados (Hair et al., 2005; Mingoti, 2005; Santana, 2006). A matriz 
de cargas fatoriais, que mede a correlação entre os fatores comuns e as variáveis observáveis, é determinada por meio da matriz de correlação, conforme Dillon e Goldstein (1984).

Na definição do Índice de Desempenho Competitivo (IDC), conforme Santana (2006), estimou-se a matriz de escores fatoriais após a rotação ortogonal da estrutura fatorial inicial. O escore fatorial, por definição, situa cada observação no espaço dos fatores comuns. Para cada fator $\boldsymbol{f}_{j}$, o i-ésimo escore fatorial extraído é definido por $\boldsymbol{F}_{i j}$, expresso da seguinte forma (Dillon; Goldstein, 1984; SPSS, 1997):

$$
F_{i j}=b_{1} x_{i 1}+b_{2} x_{i 2}+\ldots+b_{p} x_{i p} ; i=1,2, \ldots, n ; j=1,2, \ldots, p
$$

em que $\boldsymbol{b}_{\boldsymbol{i}}$ são os coeficientes de regressão estimados para os $n$ escores fatoriais comuns e $\boldsymbol{x}_{i j}$ são as $n$ observações das $p$ variáveis observáveis.

A variável $\boldsymbol{F}_{i j}$ não é observável, mas pode ser estimada por meio das técnicas de análise fatorial, utilizando-se a matriz de observações do vetor $\boldsymbol{x}$ de variáveis observáveis. Em notação matricial, a equação 2 torna-se:

$$
F_{(n, q)}=x_{(n, p)} b_{(p, q)}
$$

Na equação 3, $\boldsymbol{F}$ é a matriz da regressão estimada a partir dos $n$ escores fatoriais, os quais podem ser afetados tanto pela magnitude quanto pelas unidades de medida das variáveis $\boldsymbol{x}$. Para contornar esse tipo de problema, substitui-se a variável $\boldsymbol{x}$ pela variável padronizada $\boldsymbol{w}$, dada pela razão entre o desvio em torno da média e o desvio-padrão de $\boldsymbol{x}$, como a seguir:

$$
w_{i j}=\frac{\left(x_{i}-\bar{x}\right)}{S_{x}}
$$

Com esses valores, modifica-se a equação 3 para gerar a equação 4 . 
Zilda Joaquina Cohen Gama, Antônio Cordeiro de Santana,

Fernando Antônio Teixeira Mendes \& Ahmad Saeed Khan

$$
F_{(n, q)}=\mathcal{w}_{(n, p)} \beta_{(p, q)}
$$

Na equação 4, a matriz de pesos beta - $\boldsymbol{\beta}$, com $\boldsymbol{q}$ colunas e $\boldsymbol{p}$ coeficientes de regressão padronizados, substitui $\boldsymbol{b}$, visto que as variáveis estão padronizadas em ambos os lados da equação. Pré-multiplicando ambos os lados da equação 4 pelo valor $(\mathbf{1} / \mathbf{n}) \boldsymbol{w}^{\prime}$, em que n é o número de observações e $\boldsymbol{w}$ 'é a matriz transposta de $\boldsymbol{w}$, obtém-se:

$$
\frac{1}{n} w_{(p, n)}^{\prime} F_{(n, q)}=\frac{1}{n} w_{(p, n)}^{\prime} w_{(n, p)} \beta_{(p, q)}=R_{(p, r}
$$

A matriz $(1 / k) w^{\prime} w$ se constitui na matriz de variáveis intercorrelacionadas ou matriz de correlação entre as observações da matriz $\boldsymbol{x}$, designada por $\boldsymbol{R}$. A matriz $(\mathbf{1} / \boldsymbol{k}) \boldsymbol{w}^{\prime} \boldsymbol{F}$ representa a correlação entre os escores fatoriais e os próprios fatores, denotada por $\boldsymbol{\Lambda}$. Reescrevendo a equação 5 , temse:

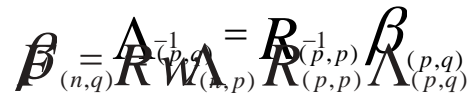

Se a matriz $\boldsymbol{R}$ for não-singular, pode-se pré-multiplicar ambos os lados da equação 6 pela inversa de $\boldsymbol{R}$, obtendo-se:

Substituindo o vetor $\boldsymbol{\beta}$ na equação 4 , obtém-se o escore fatorial associado a cada observação, como a seguir:

O IDC é definido como uma combinação linear desses escores fatoriais e a proporção da variância explicada por cada fator em relação à variância comum. A expressão matemática é dada por: 


$$
I D C_{i}=\sum_{j=1}^{q}\left(\frac{\lambda_{j}}{\sum_{j} \lambda_{j}} F P_{i j}\right),(i=1,2, \ldots, n)
$$

em que $\lambda$ é a variância explicada por cada fator e $\Sigma \lambda$ é a soma total da variância explicada pelo conjunto de fatores comuns. $\mathrm{O}$ escore fatorial foi padronizado $(F P)$ para obter valores positivos dos escores originais $\mathrm{e}$ permitir a hierarquização das empresas, uma vez que os valores do IDC estão situados entre zero e 1 . A fórmula é a seguinte:

$$
F P_{i}=\left(\frac{F_{i}-F_{\text {min }}}{F_{\text {max }}-F_{\text {min }}}\right)
$$

em que $\boldsymbol{F}_{\text {min }}$ e $\boldsymbol{F}_{\text {max }}$ são os valores máximo e mínimo observados para os escores fatoriais associados às empresas de móveis de madeira.

Valores do IDC iguais ou superiores a 0,70 são considerados altos, visto que a empresa conseguiu harmonizar as forças de seu ambiente competitivo, representando um salto do nível intermediário para grau de liderança competitiva; valores situados entre 0,4 e 0,69 são intermediários, o que caracteriza um estádio de conformismo da empresa, e mover-se daí para um patamar de liderança requer um choque de competitividade sistêmica; valores inferiores a 0,4 são considerados baixos e contemplam as empresas seguidoras com dificuldade de criar vantagens competitivas sustentáveis.

\subsubsection{Dados e variáveis}

Os dados utilizados na pesquisa são oriundos de pesquisa de campo, realizada em empresas de móveis da RMB em 2003, no âmbito da pesquisa "Organização e Competitividade da Indústria de Móveis do Pará, 2003" (Carvalho; Santana, 2005). 
Zilda Joaquina Cohen Gama, Antônio Cordeiro de Santana,

Fernando Antônio Teixeira Mendes \& Ahmad Saeed Khan

As empresas entrevistadas foram eleitas com base nos cadastros do Serviço Brasileiro de Apoio às Micro e Pequenas Empresas (Sebrae) e da Federação da Indústria do Estado do Pará (Fiepa). Uma amostra aleatória simples de 53 empresas foi extraída do universo de 337 empresas em 2003, com nível de confiança de $95 \%$ e erro amostral de 1,94, conforme descrito em Santana e Carvalho (2005). Com base nesse critério, foram entrevistadas 45 microempresas e 8 pequenas empresas. $\mathrm{O}$ instrumento de coleta dos dados foi um questionário preparado para a indústria de móveis, combinando e adaptando questões contidas no questionário utilizado para competitividade sistêmica de Coutinho e Ferraz (1994) e Santana (2002).

Para este trabalho, foram eleitas variáveis diretamente associadas às forças competitivas, delineadas nos modelos teóricos de cinco forças competitivas de Porter, de estrutura-conduta-desempenho e de competitividade sistêmica.

As variáveis escolhidas para análise foram: V1 - organização da empresa em departamentos; V2 - subcontratação da produção ou de serviços por parte das empresas; V3 - nível de capacidade ociosa das empresas; V4 - acesso a crédito de curto prazo, para capital de giro e comercialização; V5 - acesso a crédito de longo prazo, para investimento; V6 - total de pessoas ocupadas nas empresas; V7 - salário médio pago às pessoas ocupadas; V8 - qualificação da mão-de-obra, contemplando os funcionários com instrução maior ou igual ao ensino fundamental; V9 - indicador de inovação tecnológica da empresa, considerando a média de tempo de uso das máquinas, utilização de design e novas linhas de produtos, tratamento de resíduos, utilização de normas técnicas da série ISO 14.000 e técnicas de organização da produção; V10 - presença e acesso a assistência técnica; V11 - normas técnicas utilizadas para produto e para o processo como um todo; V12 - tempo que a empresa vende para o mercado nacional e internacional; V13 - clientes, incluindo os fatores que favorecem a escolha dos clientes; V14 - número de fornecedores de matérias-primas e insumos; V15 - critério de escolha do fornecedor, envolvendo preço, qualidade dos produtos, pontualidade, 
prazo de pagamento, porte do fornecedor; V16 - market share da empresa no mercado de móveis paraense; e V17 - margem de lucro em relação ao custo total obtido pela empresa. Essas variáveis constam nas dimensões consideradas como determinantes da competitividade empresarial dos modelos estrutura-conduta-desempenho (ECD), das cinco forças de Porter (CFP) e da competitividade sistêmica (CS). Como essas variáveis foram arranjadas de forma diferente em cada modelo, não se espera obter fatores expressando um modelo específico, mas dimensões competitivas em geral.

Nota-se que o número de variáveis é considerado grande em relação ao tamanho da amostra, que conta com apenas 53 empresas, numa razão de três observações para cada variável. A priori, o recomendado seria a utilização de pelo menos cinco observações para cada variável. Diante desse problema, foram feitos testes para aferir a adequação do método à amostra de dados. Os dois principais testes aplicados foram: teste de esfericidade de Bartlett, que avalia a significância geral da matriz de correlação, ou seja, testa a hipótese nula de que a matriz de correlação é uma matriz identidade; e teste de Kaiser-Meyer-Olkin (KMO), que se baseia no princípio de que a inversa da matriz de correlação se aproxima da matriz diagonal e, para tanto, compara as correlações entre as variáveis observáveis.

As fórmulas matemáticas desses testes são as seguintes (Dillon; Goldstein, 1984; Reis, 2001; Mingoti, 2005):

$$
K M O=\frac{\sum_{i} \sum_{j} r_{i j}^{2}}{\sum_{i} \sum_{j} r_{i j}^{2}+\sum_{i} \sum_{j} a_{i j}^{2}}
$$

em que $\boldsymbol{r}_{i j}$ é o coeficiente de correlação da amostra entre as variáveis $\boldsymbol{x}_{\boldsymbol{i}}$ e $\boldsymbol{x}_{\boldsymbol{j}}$ e $\boldsymbol{a}_{i j}$ é o coeficiente de correlação parcial entre as mesmas variáveis, que é, simultaneamente, uma estimativa das correlações entre os fatores, eliminando o efeito das demais variáveis. Os $\boldsymbol{a}_{i j}$ deverão assumir valores 
Zilda Joaquina Cohen Gama, Antônio Cordeiro de Santana,

Fernando Antônio Teixeira Mendes \& Ahmad Saeed Khan

próximos de zero, uma vez que se pressupõe que os fatores são ortogonais entre si. Valores deste teste abaixo de 0,50 são inaceitáveis (Hair et al., 2005).

O teste de Bartlett de esfericidade testa a hipótese nula de que as variáveis são independentes, contra a hipótese alternativa de que as variáveis são correlacionadas entre si. Ou seja: $\mathrm{H}_{0}: \boldsymbol{R}=\boldsymbol{I}$ ou $\mathrm{H}_{0}: \lambda_{1}=\lambda_{2}$ $=\ldots=\lambda_{\mathrm{p}}$, é dado pela equação 11 :

$$
\begin{aligned}
& \chi^{2}=-\left[n-1-\frac{1}{6}(2 p+5)\right] \cdot \ln |R| \mathrm{ou} \\
& \chi^{2}=-\left[n-1-\frac{1}{6}(2 p+5)\right] \cdot \sum_{i=1}^{p} \ln \lambda_{i}
\end{aligned}
$$

em que $|\boldsymbol{R}|$ é o determinante da matriz de correlação da amostra; $\lambda$, a variância explicada por cada fator; $\boldsymbol{n}$, o número de observações; e $\boldsymbol{p}, \mathrm{o}$ número de variáveis. A estatística tem uma distribuição assintótica de $\chi^{2}$ com $[0,5 . p .(p-1)]$ graus de liberdade.

\section{Resultados}

O teste $\mathrm{KMO}$ foi da ordem de 0,533 , indicando adequação da amostra à análise fatorial. O teste de Bartlett, com valor de 316,681, significante a $1 \%$, permitiu aceitar a hipótese alternativa de que as variáveis são correlacionadas. Portanto, a significância dos testes atesta a adequação da amostra de dados ao modelo de análise fatorial.

Pelo critério da raiz latente, o modelo elegeu seis fatores de competitividade entre as 17 variáveis analisadas. Esses fatores explicaram $67,93 \%$ da variância total (Tabela 1 ). 
A matriz de cargas fatoriais rotacionada foi apresentada na Tabela 2. Nesta tabela foram identificadas as variáveis que compõem cada um dos fatores, bem como o grau de correspondência (correlação) entre cada variável e cada fator. A comunalidade, que resulta da soma das cargas fatoriais ao quadrado de cada linha e indica o quanto da variância de cada variável foi explicado pelo conjunto de fatores, também consta na Tabela 2.

Tabela 1 - Total da variância explicada dos fatores obtidos através da análise fatorial das empresas de móveis da RMB, 2003

\begin{tabular}{cccccccc}
\cline { 2 - 7 } Fatores & \multicolumn{3}{c}{ Autovalores iniciais } & \multicolumn{4}{c}{ Variâncias iniciais } \\
\cline { 2 - 7 } & Total & $\begin{array}{c}\text { \% da } \\
\text { Variância }\end{array}$ & $\begin{array}{c}\text { Acumulado } \\
\%\end{array}$ & Total & $\begin{array}{c}\text { \% da } \\
\text { Variância }\end{array}$ & Acumulado & Tota \\
\hline 1 & 3,501 & 20,592 & 20,592 & 3,501 & 20,592 & 20,592 & 3,11 \\
2 & 2,300 & 13,531 & 34,124 & 2,300 & 13,531 & 34,124 & 2,06 \\
3 & 1,768 & 10,4 & 44,524 & 1,768 & 10,4 & 44,524 & 1,74 \\
4 & 1,364 & 8,023 & 52,547 & 1,364 & 8,023 & 52,547 & 1,73 \\
5 & 1,319 & 7,761 & 60,308 & 1,319 & 7,761 & 60,308 & 1,49 \\
6 & 1,295 & 7,619 & 67,927 & 1,295 & 7,619 & 67,927 & 1,3 \\
7 & 0,996 & 5,859 & 73,786 & & & & \\
8 & 0,862 & 5,071 & 78,857 & & & & \\
9 & 0,734 & 4,319 & 83,176 & & & & \\
10 & 0,722 & 4,248 & 87,425 & & & & \\
11 & 0,6 & 3,532 & 90,957 & & & & \\
12 & 0,428 & 2,52 & 93,477 & & & & \\
13 & 0,352 & 2,07 & 95,547 & & & & \\
14 & 0,322 & 1,893 & 97,439 & & & & \\
15 & 0,242 & 1,426 & 98,865 & & & & \\
16 & 0,138 & 0,81 & 99,675 & & & & \\
17 & 0,055 & 0,325 & 100 & & & & \\
\hline
\end{tabular}

Fonte: Resultados da pesquisa.

As variáveis que têm maior peso na explicação dos seis fatores de competitividade das empresas de móveis da RMB foram: V8 - índice de qualificação da mão-de-obra (87,3\%), V16 - market share (85,5\%), V2 - subcontratação da produção ou de serviços $(79,1 \%)$, V5 - obtenção de crédito de longo prazo $(77,5 \%), \mathrm{V} 4$ - obtenção de crédito de curto prazo (76,9\%), V12 - tempo de venda para o mercado nacional $(75,4 \%), \mathrm{V} 14$ - número de fornecedores $(73,9 \%)$ e V15 - critério de escolha do fornecedor $(71,9 \%)$. As variáveis com comunalidades pequenas (V7 - 
Zilda Joaquina Cohen Gama, Antônio Cordeiro de Santana,

Fernando Antônio Teixeira Mendes \& Ahmad Saeed Khan

salário médio e V9 - indicador de tecnologia) mostram que boa parte da variância dessas variáveis não foi explicada pelos fatores (Tabela 2).

A escolha das variáveis que compõem cada um dos seis fatores se deu observando as cargas fatoriais de cada variável, da esquerda para direita e ao longo de cada linha, elegendo-se a carga fatorial de maior valor absoluto.

O primeiro fator, denominado de gestão empresarial, explicou $18,32 \%$ da variância total dos dados e englobou as seguintes variáveis: V6 total de pessoas ocupadas, V14 - número de fornecedores, V1 organização em departamentos, V16 - market share, V7 - salário médio e V8 - índice de qualificação da mão-de-obra (Tabela 2). As variáveis V6, V7 e V8, todas relacionadas aos recursos humanos (quantidade e qualidade), apresentaram relação positiva com esse fator. Essas variáveis são fontes de vantagens competitivas tanto de custo (salários baixos) quanto de diferenciação (mão-de-obra qualificada) para as empresas. Neste fator constam variáveis consideradas nas três dimensões do modelo ECD, no nível micro da competitividade sistêmica e nos ambientes de rivalidade e de gestão interna do modelo de Porter. 
REVISTA DE ECONOMIA E AGRONEGÓCIO, VOL.5, $N^{o} 1$ 
Zilda Joaquina Cohen Gama, Antônio Cordeiro de Santana,

Fernando Antônio Teixeira Mendes \& Ahmad Saeed Khan

A variável V8 (índice de qualificação da mão-de-obra) apresentou carga fatorial elevada, demonstrando ser uma importante fonte de vantagem competitiva para as empresas de móveis da RMB. Considerando-se que o percentual de funcionários analfabetos ou com primário incompleto é elevado e a importância dessa variável para a criação de vantagens competitivas, o diferencial competitivo está com as empresas que apresentam o maior número de funcionários qualificados ou aptos para executar tarefas e funções diferenciadas na empresa. Esta variável é um importante indicador de competitividade para os três modelos de referência, uma vez que contribui para aumentar a qualidade e diferenciação dos produtos e para redução do custo médio mediante efeito da curva de aprendizado.

A variável salário médio (V7) apresentou carga fatorial baixa $(0,401)$, portanto com pequena influência sobre a gestão competitiva das empresas de móveis da RMB. Ou seja, as empresas que se pautam nesta variável como estratégia principal podem não sobreviver no mercado em longo prazo. Esta variável é considerada na dimensão conduta do modelo ECD e nível micro do modelo de competitividade sistêmica.

O market share (V16) também está relacionado positivamente ao primeiro fator, apresentando a maior carga fatorial $(0,885)$. Um dos principais objetivos de crescimento revelado pelos empresários foi o desejo de ampliar a parcela de mercado (Carvalho; Santana, 2005). Em tese, quanto maior for a parcela de participação no mercado da empresa, maior tende a ser sua importância e força competitiva entre as empresas rivais, uma vez que atende maior parcela dos consumidores segundo suas exigências em termos de qualidade, diversificação e diferenciação de produtos e pronto atendimento. Esta variável é um indicador de desempenho do modelo ECD e variável resultado para os modelos de Porter e de competitividade sistêmica.

A variável V14 (número de fornecedores) relacionada a uma das principais forças competitivas abordadas por Porter (1993), apresentou relação positiva com a dimensão gestão empresarial, indicando que boas relações com os fornecedores implicam efeitos positivos sobre o 
primeiro fator determinante da competitividade. Esta variável é considerada na dimensão estrutura do modelo ECD, no poder dos fornecedores no modelo de Porter e no nível micro do modelo de competitividade sistêmica.

Por outro lado, a variável V1 (organização da empresa em departamentos) apresentou relação negativa com a dimensão gestão empresarial, em razão do reduzido número de empresas que iniciaram sua estrutura departamental e contam apenas com o departamento de recursos humanos; por isso, os reflexos positivos dessa organização ainda não são percebidos como determinantes de vantagens competitivas nessas empresas (ambiente conduta no modelo ECD, micro no modelo de competitividade sistêmica e rivalidade no modelo de Porter).

Esse primeiro fator, à luz dos modelos de competitividade utilizados como referência, não aponta para o nível de desenvolvimento industrial para o qual tais modelos foram criados. Sinaliza para uma visão sistêmica, incluindo variáveis indicadoras dos pontos de ameaça ao crescimento das empresas de móveis da RMB.

O segundo fator, denominado dimensão tecnológica, foi definido pelas variáveis V10 - presença de assistência técnica, V13 - fator que favorece a escolha do cliente (qualidade do produto, preço, estilo) e V9- indicador de inovação tecnológica de produto, processo e de gestão empresarial, bem como os aspectos ambientais de tratamento de resíduos e aquisição de matéria-prima certificada e/ou de floresta plantada. Este fator contempla variáveis da dimensão conduta do modelo ECD, dos ambientes micro e mesoanalíticos da competitividade sistêmica e as estratégias do modelo de Porter. Ele explicou 12,16\% da variância total dos dados e possui relação positiva com as variáveis: assistência técnica e indicador de tecnologia (medido pela média do tempo de uso das máquinas), sinalizando para a contribuição sinérgica que essas variáveis têm na criação de vantagens competitivas para as empresas de móveis da RMB. A inovação tecnológica e de gestão possibilita a redução dos custos, aumento na qualidade dos produtos, adiciona valor e faz a adequação do 
Zilda Joaquina Cohen Gama, Antônio Cordeiro de Santana,

Fernando Antônio Teixeira Mendes \& Ahmad Saeed Khan

produto aos segmentos de mercado. Em conjunto com mão-de-obra, a empresa pode criar as vantagens competitivas por diferenciação e o aprimoramento da qualidade do produto.

O indicador de inovação tecnológica reflete a produção em série de móveis retilíneos, uso de MDF, painéis, secagem de madeira, adequação de verniz com as madeiras secas em estufa ou ao sol, utilização de ferro e vidro, o que necessita de assistência técnica para uso de máquinas, acabamento, desenho, e assim por diante.

Essa dimensão possui relação negativa com a variável indicadora dos fatores que favorecem a escolha dos clientes (preços, porte da empresa, prazo de pagamento, etc.), pois o principal fator de escolha dos clientes é o preço de venda, e o preço não constitui o melhor critério para vencer concorrência, pois essas vantagens podem ser rapidamente eliminadas pelas empresas concorrentes. Todavia, a relação negativa pode estar na incompatibilidade de se adotar tecnologia para melhorar a qualidade e diferenciar o produto com o foco em preços baixos.

Ao terceiro fator foram associadas as variáveis V4 - obtenção de crédito de curto prazo e V5 - obtenção de crédito de longo prazo. Este fator, chamando de dimensão de crédito, explicou 10,28\% da variância total dos dados. Tanto a variável V4 quanto a V5 se relacionaram positivamente com a dimensão de crédito. O crédito tem importância fundamental na geração de vantagens competitivas, uma vez que possibilita o investimento na compra de máquinas e equipamentos, qualificação da mão-de-obra, utilização de inovações tecnológicas e de gestão, prospectar o mercado e estruturar as conexões com os elos de distribuição da cadeia produtiva. No caso das empresas de móveis da RMB, entretanto, poucas tiveram acesso a crédito, em razão de dificuldades de enquadramento na burocracia inerente ao sistema de concessão de crédito nos bancos oficiais, como garantias, legalização, adequação da taxa de juros e aos prazos de pagamento. $\mathrm{O}$ crédito, juntamente com as razões vinculadas ao acesso (garantias, juros, prazo, burocracia), constitui um dos fortes elementos do nível mesoanalítico da competitividade sistêmica, pois sua adequação 
carece de uma governança especial. No modelo de Porter, o crédito vincula-se às principais dimensões e na dimensão conduta do modelo ECD.

O quarto fator, denominado dimensão de desempenho, explicou $10,17 \%$ da variância total e conta com as variáveis V3 - nível de capacidade ociosa, V17 - margem de lucro e V11 - utilização de normas técnicas de produto. O nível de capacidade ociosa e a margem de lucro estão positivamente relacionados com esse fator, indicando barreira à entrada de novas empresas no mercado e desempenho competitivo, respectivamente, pelo menos em curto prazo. A variável utilização de normas técnicas de produto ou processo, por sua vez, apresentou relação negativa com essa dimensão, pois das empresas de móveis da RMB poucas utilizam essas normas e, em razão do baixo grau de generalização (pois algumas dessas normas são exigências legais), isso acaba se refletindo em aumento de custo em relação às empresas que ainda não as utilizam ou que operam no mercado informal. Este fator contempla as variáveis de dimensão desempenho do modelo ECD, dimensão rivalidade e ameaças de novos entrantes do modelo de Porter e o microambiente de análise da competitividade sistêmica (Tabela 2).

O quinto fator, denominado integração horizontal, explicou 8,81\% da variância total dos dados e foi definido pela variável V2 - subcontratação da produção ou de serviço. A variável subcontratação da produção ou de serviços tem relação positiva com este fator, uma vez que reflete o grau de conexão para trás da empresa com suas parceiras na fabricação de um produto final. Algumas empresas subcontratam outras para fabricarem partes de um produto ou o produto acabado, a fim de atender aos clientes. São casos restritos, mas que funcionam como um embrião da organização empresarial, na forma de integração horizontal cooperativa entre micro e pequenas empresas. Outras empresas subcontratam apenas os serviços de contabilidade e fornecimento de refeição, por isso a baixa contribuição desse fator para o desempenho competitivo das empresas da RMB. Este fator, embora conste de apenas uma variável, constitui uma estratégia estruturante da integração horizontal de forma cooperativa, 
Zilda Joaquina Cohen Gama, Antônio Cordeiro de Santana,

Fernando Antônio Teixeira Mendes \& Ahmad Saeed Khan

fundamental para neutralizar as ameaças de novos entrantes e a ação de rivais de acordo com Porter, assim como fortalece o tecido empresarial para dialogar com as instâncias institucionais do nível mesoanalítico da competitividade sistêmica. Além disso, os resultados do modelo fatorial respaldam sua inclusão, pois este apresenta alto valor da diagonal principal de correlação antiimagem $(0,494)$, comunalidade $(0,791)$ e peso $(0,861)$.

O sexto fator, denominado conexão de mercado, explicou apenas $8,18 \%$ da variância total dos dados e conta com as variáveis V15 - critério de escolha do fornecedor, que reflete os indicadores de maior importância para as empresas, como preço da matéria-prima e insumos, qualidade do produto, regularidade na entrega e prazo para pagamento; e V12 - tempo de venda para o mercado nacional, abrangendo assim o relacionamento com fornecedores e clientes, por meio de contratos formais e/ou informais e acesso a mercado. Este fator está contemplado na dimensão estrutura do modelo ECD, no nível micro da competitividade sistêmica e nos ambientes de poder do fornecedor e de rivalidade do modelo de Porter; como no fator 5 , os resultados estatísticos respaldam sua inclusão no modelo.

Os escores fatoriais associados a cada um dos fatores utilizados para estimar o IDC e os pesos (betas) foram apresentados na Tabela 3. 
Tabela 3 - Matriz de pesos dos escores fatoriais das empresas de móveis da RMB

\begin{tabular}{ccccc} 
& \multicolumn{3}{c}{ Variáveis } & \multicolumn{3}{c}{ Fatores } \\
\cline { 2 - 5 } & F1 & F2 & F3 & F4 \\
\hline V6 & 0,1202 & 0,1661 & $-0,2329$ & $-0,1604$ \\
V2 & $-0,035$ & 0,0031 & $-0,012$ & 0,000 \\
V3 & $-0,0225$ & 0,0276 & 0,0446 & 0,4386 \\
V4 & 0,1243 & 0,0073 & 0,5353 & 0,073 \\
V5 & 0,0528 & 0,0313 & 0,4803 & $-0,0325$ \\
V10 & 0,0309 & 0,3112 & $-0,0214$ & 0,1067 \\
V14 & 0,2477 & 0,2212 & 0,0936 & $-0,2304$ \\
V15 & $-0,0913$ & $-0,0341$ & $-0,1651$ & 0,1523 \\
V12 & 0,0219 & 0,0249 & 0,0945 & $-0,1764$ \\
V17 & 0,0126 & 0,0468 & 0,0377 & 0,3992 \\
V13 & 0,0344 & $-0,3704$ & 0,0442 & $-0,0817$ \\
V11 & $-0,1302$ & 0,2428 & $-0,0262$ & $-0,2311$ \\
V1 & $-0,2134$ & 0,0502 & 0,0371 & 0,058 \\
V16 & 0,2971 & $-0,0388$ & 0,0829 & 0,0712 \\
V7 & 0,1326 & $-0,1882$ & $-0,0017$ & 0,1611 \\
V8 & 0,3012 & $-0,0153$ & 0,1364 & 0,088 \\
V9 & 0,0206 & 0,2982 & 0,0622 & 0,0162 \\
\hline
\end{tabular}

Fonte: Resultados da pesquisa.

Os resultados mostram que apenas uma empresa apresentou alto desempenho competitivo (IDC $=0,7108$ ). Esta empresa obteve sinais positivos e altos nos quatro mais importantes escores fatoriais e sinal negativo nos dois últimos. Os escores fatoriais positivos nas dimensões gestão empresarial, tecnológica, crédito e desempenho indicam que a empresa está conseguindo orientá-los para a criação de vantagens competitivas sustentáveis, muito embora apresente fraca integração com 
Zilda Joaquina Cohen Gama, Antônio Cordeiro de Santana,

Fernando Antônio Teixeira Mendes \& Ahmad Saeed Khan

fornecedores e clientes. Ou seja, o alto desempenho competitivo dessa empresa só foi possível porque ela possui mão-de-obra qualificada, recebeu assistência técnica, possui máquinas com até dois anos de uso e teve acesso a crédito de curto e longo prazo, porém o seu desempenho não foi maior porque apresentou deficiências na relação com fornecedores e clientes e não possui empresas subcontratadas, especializadas em dada etapa do processo produtivo (Tabela 4).

Somente quatro empresas apresentaram IDC inferior a 0,4 , ou seja, baixo desempenho competitivo. Em três dessas empresas, existe apenas uma dimensão com escore fatorial positivo, e em uma delas todas as dimensões fatoriais foram negativas. Isso revela um quadro de baixa capacidade de gestão empresarial dessas empresas.

Deficiências comuns a essas empresas estão relacionadas aos escores fatoriais negativos em algumas dimensões, em função dos seguintes aspectos: insignificante preocupação com a qualificação da mão-de-obra; baixo nível do salário médio; não-utilização de normas técnicas de produto e/ou processo; insignificante grau de subcontratação de empresas; deficiência nos critérios de escolha do fornecedor; curto período de tempo na venda para o mercado nacional; ausência de demanda ou inexistência de assistência técnica; elevado tempo de uso das máquinas; e tecnologia ultrapassada.

A grande maioria das empresas mostrou desempenho competitivo intermediário (IDC entre 0,7 e 0,4), e 55,8\% apresentaram IDC acima do valor médio de 0,5093 .

A segunda empresa obteve IDC de 0,6377 , com valores positivos em todos os escores fatoriais, exceto na dimensão gestão empresarial, que se revelou a mais importante para definir as estratégias competitivas criadas pelas empresas de móveis da RMB. Por essa razão, ela não alcançou alto desempenho competitivo. 
Das empresas com índice de desempenho competitivo intermediário, duas obtiveram sinal positivo nos escores de todas as dimensões, indicando que as estratégias criadas por elas estão sistemicamente atingindo todas as dimensões, porém é necessário maior força para impulsioná-las a alcançarem a posição de liderança. 
Zilda Joaquina Cohen Gama, Antônio Cordeiro de Santana,

Fernando Antônio Teixeira Mendes \& Ahmad Saeed Khan

Tabela 4 - Valores dos escores fatoriais originais e padronizados e o índice de desempenho competitivo das empresas de móveis da RMB

\begin{tabular}{|c|c|c|c|c|c|c|c|c|}
\hline \multirow{2}{*}{ Empresa } & \multicolumn{6}{|c|}{ Escore fatorial original } & \multicolumn{2}{|r|}{$\mathbf{E}$} \\
\hline & F1 & F2 & F3 & F4 & F5 & F6 & Fp1 & $\overline{F p}$ \\
\hline Maso Industrial S/A & 5,4077 & 0,6143 & 0,4923 & 0,3848 & $-2,2578$ & $-0,5797$ & 1 & 0,71 \\
\hline F. de Sousa Carvalho Madeira & $-0,0881$ & 0,3928 & 0,928 & 1,6458 & 0,5982 & 0,9232 & 0,1466 & $0,6^{\circ}$ \\
\hline Americo F. Silva Indústria e Comércio & 0,2086 & 0,5772 & 0,1966 & 1,6322 & $-0,4801$ & 2,3986 & 0,1926 & 0,71 \\
\hline G. N. Souto M.E. & 0,1139 & 0,1712 & 0,7823 & 1,3759 & 0,7995 & 0,5816 & 0,1779 & 0,6 \\
\hline Perfini Indústria e Comércio Ltda & 2,1799 & 0,4859 & 0,6099 & $-0,9435$ & $-0,1705$ & 0,7492 & 0,4988 & $0,6 i$ \\
\hline Urnas Mart Ltda & 1,4258 & 0,9518 & $-0,3624$ & $-0,3889$ & 0,6856 & 0,5377 & 0,3816 & 0,7 \\
\hline Marcenaria e Carpintaria Mimóveis & $-0,2029$ & 0,8852 & 0,8592 & 0,7931 & 0,4058 & 0,4123 & 0,1287 & $0,7:$ \\
\hline Marcenaria Caio Móveis & 0,139 & 0,3196 & 0,9433 & 1,1231 & 0,1105 & 0,2397 & 0,1818 & $0,6 t$ \\
\hline Benção de Deus & 0,0986 & 0,5791 & 0,6716 & $-0,3163$ & 0,8256 & 0,2985 & 0,1755 & 0,71 \\
\hline Movelaria São Pedro & $-0,2809$ & 0,5876 & 0,7007 & 0,0365 & 0,5063 & 0,5301 & 0,1166 & 0,71 \\
\hline Não identificado pela pesquisa & $-0,5356$ & 0,5322 & 0,7449 & 0,969 & 0,0906 & 0,1 & 0,0771 & 0,6 ? \\
\hline Ravel Kadela Indústria & $-0,1827$ & 0,8008 & 0,7204 & 1,2606 & 0,5949 & $-1,7708$ & 0,1319 & 0,7 . \\
\hline Reginaldo S. Almeida & $-0,4868$ & 0,6692 & 0,3611 & 0,7697 & $-0,267$ & 0,7275 & 0,0846 & 0,7 \\
\hline Carpintaria São Jorge & $-0,1583$ & 0,2539 & 0,6937 & $-0,4584$ & 0,6044 & 0,6324 & 0,1357 & 0,6 : \\
\hline S.A. Moreira e Meira & $-0,1595$ & $-1,1641$ & 0,6202 & 0,2873 & 0,7852 & 1,0117 & 0,1355 & 0,4 \\
\hline Tapanã LTDA & 1,5311 & $-1,1333$ & $-1,4605$ & 1,4179 & 0,6155 & $-0,3406$ & 0,398 & 0,4 : \\
\hline Orivaldo Frota Pereira & $-0,2837$ & 0,3807 & 0,7027 & $-0,5395$ & 0,4204 & 0,5338 & 0,1162 & $0,6^{\circ}$ \\
\hline Mad Móveis & 0,1573 & 0,2329 & 0,448 & 0,3157 & 0,5505 & $-0,9943$ & 0,1847 & 0,6 \\
\hline Lambari Compensados & $-0,432$ & 0,3436 & 0,5219 & 1,4171 & $-0,0354$ & $-1,0557$ & 0,0931 & $0,6 t$ \\
\hline Belmodulo & 1,1938 & 2,3962 & $-0,7456$ & $-3,1923$ & 1,0894 & 0,1924 & 0,3456 & \\
\hline Movelaria Santo Antonio & $-0,5074$ & 0,3338 & 0,5167 & 1,4562 & $-0,064$ & $-1,0928$ & 0,0814 & $0,6 t$ \\
\hline Maria S. Pereira & 0,3325 & $-1,7909$ & 0,6333 & $-0,039$ & 1,2352 & 0,4138 & 0,2119 & 0,3 \\
\hline Móveis e Decorações Carvalho Ltda & $-0,3407$ & 0,399 & 0,3546 & 1,1817 & $-0,1324$ & $-1,0884$ & 0,1073 & $0,6^{*}$ \\
\hline Maria Pinto Coelho & $-0,4526$ & 0,5635 & $-0,1283$ & $-0,0108$ & 0,2246 & 0,7451 & 0,09 & 0,7 \\
\hline J. Atamazio Ind. Com. e Exp. Ltda & 0,3923 & $-0,3942$ & 0,4869 & 0,1199 & 0,602 & $-1,2184$ & 0,2212 & 0,5 \\
\hline Hamilton Vanzeler & $-0,273$ & $-0,3986$ & 0,6926 & $-0,4352$ & 0,4653 & 0,4802 & 0,1178 & 0,5 \\
\hline Toraleys Exp. Ltda & 0,0417 & 0,8695 & $-0,1071$ & $-1,1976$ & 0,5544 & 0,3769 & 0,1667 & $0,7:$ \\
\hline Oficina Globo de Ouro & $-0,5481$ & 0,2281 & 0,6009 & $-0,0528$ & 0,1602 & $-0,0398$ & 0,0751 & 0,6 \\
\hline Indústria de Móveis Austrália Ltda & 0,029 & 0,3807 & $-0,6926$ & $-0,9338$ & 0,596 & 1,3116 & 0,1647 & $0,6^{\circ}$ \\
\hline Marcenaria Santos & $-0,4553$ & 0,252 & 0,5113 & $-0,3709$ & 0,216 & $-0,2767$ & 0,0895 & 0,6 : \\
\hline Gab Móveis & $-0,5909$ & 0,2603 & 0,5778 & 0,3868 & $-0,0031$ & $-1,1531$ & 0,0685 & 0,6 : \\
\hline Movelaria Campos & $-0,7433$ & 0,4625 & $-1,0649$ & 0,9675 & $-0,1445$ & 0,5297 & 0,0448 & 0,6 \\
\hline Massa Móveis Ltda & 1,1615 & $-1,5963$ & $-1,556$ & 0,6075 & 1,0647 & $-0,606$ & 0,3406 & 0,3 \\
\hline Moraes Móveis & $-0,4829$ & 0,1943 & 0,4502 & $-0,0424$ & 0,006 & $-0,8798$ & 0,0852 & 0,6 \\
\hline Marcenaria Eucalipal & $-0,5726$ & 0,4617 & 0,551 & $-1,3778$ & 0,2556 & 0,2086 & 0,0713 & $0,6 i$ \\
\hline A. C. Vargas & $-0,1547$ & $-0,0099$ & 0,5464 & $-1,2156$ & $-0,0531$ & $-0,0567$ & 0,1362 & 0,61 \\
\hline Marceneria Fé em Deus & $-0,2038$ & 0,182 & $-0,7577$ & $-0,5842$ & 0,949 & $-0,2354$ & 0,1286 & 0,6 \\
\hline A. Sampaio & $-0,645$ & $-0,6147$ & 0,5948 & $-0,7507$ & 0,263 & 0,0277 & 0,0601 & 0,51 \\
\hline Não identificado na pesquisa & $-1,0318$ & 0,4308 & $-1,9609$ & 1,3466 & $-2,9156$ & 3,32 & 0 & $0,6^{\circ}$ \\
\hline N. A. Veiga Sampaio Ltda & 1,0087 & $-2,5834$ & $-1,5736$ & 0,6761 & 0,3117 & $-0,1385$ & 0,3169 & $0,1 i$ \\
\hline R. E. Alves da Silva & $-0,276$ & $-0,6319$ & 0,8186 & $-1,164$ & $-1,6936$ & 0,4199 & 0,1174 & 0,51 \\
\hline Meirelhes e Meira I tda & ก ก861 & -12803 & -18600 & ก 5496 & 09743 & $-n 8775$ & 01736 & $n 41$ \\
\hline
\end{tabular}

Fonte: Resultados da pesquisa. 
Por fim, cabe ressaltar que as empresas que apresentaram IDC intermediário encontram-se numa posição desconfortável, pois chegaram a esse nível construindo vantagens de custos. A evolução para o estágio de alto desempenho competitivo somente se dará através da diferenciação, o que requer investimentos em tecnologia, capacitação da mão-de-obra, pesquisas de mercado, implantação de normas técnicas de processo e produto e construção das conexões para frente e para trás. Esse salto é difícil de ser alcançado, em face do comportamento oportunista dos agentes e instituições, da dependência tecnológica, da baixa integração para frente e do incipiente grau de organização empresarial.

\section{Conclusões}

A análise fatorial agrupou as variáveis que influenciam o desempenho competitivo das empresas de móveis da Região Metropolitana de Belém em seis dimensões competitivas, explicando $67,93 \%$ da variância total dos dados. Os testes de Kaiser-Meier-Olkin e de Bartlett respaldaram a adequação da amostra de dados ao modelo de análise fatorial.

Os resultados permitiram a extração de seis fatores, representativos das dimensões determinantes da competitividade das empresas de móveis da RMB: gestão empresarial, envolvendo seis variáveis relacionadas com o ambiente competitivo interno das empresas; tecnológica, composta por três variáveis indicadoras das ações inovadoras de produto, processo e de gestão implantadas nas empresas; crédito, com duas variáveis que refletem a dinâmica de acesso em função de fatores relacionados a juros, garantias e adequação a prazos; desempenho, contemplando três variáveis indicadoras do desempenho competitivo das empresas de móveis; integração horizontal, com uma variável indicadora das relações de subcontratação de empresas para o desenvolvimento de peças e de produtos acabados; e conexão de mercado, envolvendo duas variáveis relacionadas ao fornecimento de matéria-prima e insumos e à inserção das empresas nos mercados. Essas dimensões apresentaram-se 
Zilda Joaquina Cohen Gama, Antônio Cordeiro de Santana,

Fernando Antônio Teixeira Mendes \& Ahmad Saeed Khan

consistentes com os modelos teóricos da competitividade sistêmica e das forças sinérgicas porterianas.

Com base nos resultados obtidos para o Índice de Desempenho Competitivo, conclui-se que a maior parte das empresas de móveis da Região Metropolitana de Belém está operando em estádio intermediário de desenvolvimento competitivo, em razão da baixa magnitude dos escores fatoriais relativos a cada dimensão competitiva e/ou de sinal negativo. Isso permite que os formuladores de política ou de fomento ao desenvolvimento industrial identifiquem as fragilidades, os pontos fortes e as oportunidades de mercado para cada empresa.

Apenas uma empresa obteve alto Índice de Desempenho Competitivo. Esta empresa pode servir de base para a articulação empresarial no seu entorno, ao estilo da integração do tipo núcleo satélite, visando disseminar estratégias competitivas para as demais empresas de móveis, bem como quebrar a inércia das estratégias pautadas apenas nas vantagens de custo, mão-de-obra informal, matéria-prima de baixa qualidade, comportamento oportunista dos agentes e concorrência focada em preço.

As dimensões competitivas eleitas conferem maior aderência às forças competitivas tradicionais, em função do estádio de desenvolvimento da indústria de móveis local. A criação de vantagens competitivas sustentáveis, com base na qualidade e diferenciação dos produtos, qualificação da mão-de-obra, inovação de processo e de gestão, bem como na organização para integração horizontal e vertical da cadeia produtiva, apenas anuncia a evolução. 


\section{Referências}

ABIMÓVEL. Panorama da indústria de móveis. Disponível em: http:/ /www.abimovel.com.br/?pg=panorama setor. Acesso em: 27 outubro de 2006.

ASSOCIAÇÃO BRASILEIRA DAS INDÚSTRIAS DO MOBILIÁRIO. Panorama do setor moveleiro no Brasil. Disponível em: <www.abimovel.com.br>. Acesso em: 4 ago 2005.

BAIN, J.S. Industrial organization. 2. ed. New York: Wiley, 1968.

BRAGA, H.C. Estrutura de mercado e desempenho da indústria brasileira: 1973/75. Rio de Janeiro: FGV, 1980. (Tese, 06).

CARVALHO, D.F.; SANTANA, A.C. de. (Coord.). Organização e competitividade da indústria de móveis do Pará. Belém: Unama, 2004. Relatório final.

CARVALHO, D.F.; SANTANA, A.C. de. Organização e competitividade da indústria de móveis do Pará. Belém: Unama, 2005.

COUTINHO, L.; FERRAZ, J.C. (Coord.). Estudo da competitividade da indústria brasileira. 2. ed. Campinas: Papirus, 1994.

DILLON, W.R.; GOLDSTEIN, M. Multivariate analysis: methods and applications. New York: John Wiley \& Sons, 1984.

ESSER, K.; HILLEBRAND, W.; MESSNER, D.; MEYER-STANNER, J. Systemic competitiveness - new challenges to business and politics. Economics, v.59, 1999.

FAIRBANKS, M.; LINDSAY, S. Arando o mar: fortalecendo as fontes ocultas do crescimento em países em desenvolvimento. Rio de Janeiro: Qualitymark Ed., 2002. 
Zilda Joaquina Cohen Gama, Antônio Cordeiro de Santana,

Fernando Antônio Teixeira Mendes \& Ahmad Saeed Khan

FARINA, E.M.M.Q.; ZILBERSZTAJN, D. (Coord.) Competitividade no agribusiness brasileiro. São Paulo: PENSA?FIA/FEA?USP, 1998.

FERRAZ, J.C.; KUPFER, D.; HAGUENAUER, L. Made in Brasil: desafios competitivos para a indústria. Rio de Janeiro: Campus, 1995.

GAMA, Zilda Joaquina Cohen. Análise da competitividade das empresas de móveis da região metropolitana de Belém, 2000 a 2004. 2006. 131 f. Dissertação (Mestrado em Economia) - Universidade da Amazônia.

GOMES, Sérgio Castro. Análise da competitividade das empresas de laminados e compensados do Estado do Pará: 1996-2001. 2003. 160 f. Dissertação (Mestrado em Economia) - Universidade da Amazônia.

HAIR JR, J.F.; ANDERSON, R.E.; TATHAM, R.L.; BLACK, W.C. Análise multivariada de dados. 5. ed. Porto Alegre: Bookman, 2005.

JOHNSON, R.A.; WICHERN, D.W. Applied multivariate statistical analysis. Prentice-Hall, 1992.

KUPFER, D. Competitividade da indústria brasileira: visão de conjunto e tendências de alguns setores, 1994. Disponível em: <www.ie.ufrj.br/gic>. Acesso em: 21 out 2004.

MINGOTI, S.A. Análise de dados através de métodos de estatística multivariada: uma abordagem aplicada. Belo Horizonte: Editora UFMG, 2005.

OCDE. Tecnology and the economy: the key relationships. Paris: OCDE, 1992.

PORTER, M. E. A vantagem competitiva das nações. Rio de Janeiro: Campus, 1993. 
PORTER, M.E. Como as forças competitivas moldam a estratégia. In: PORTER, M.E. Competição = on competition: estratégias competitivas essenciais. Rio de Janeiro: Campus, 1999. p.27-45.

POSSAS, M.L. Competitividade: fatores sistêmicos e política industrial - implicações para o Brasil. In: CASTRO, A.B.; POSSAS, M.L.; PROENÇA, A. (Org.) Estratégias empresariais na indústria brasileira: discutindo mudanças. Rio de Janeiro: Forense, 1996. P.71117.

POSSAS, M.L. Estrutura de mercado em oligopólio. 2. ed. São Paulo: Hucitec, 1987.

REIS, E. Estatística multivariada aplicada. 2. ed. Lisboa: Silabo, 2001.

SANTANA, A. C. de. A competitividade sistêmica das empresas de madeira da Região Norte. Belém: FCAP, 2002.

SANTANA, A.C. de. Análise da competitividade sistêmica da indústria de madeira no Estado do Pará. Revista de Economia e Agronegócio, Viçosa - MG, v. 1, n. 2, p. 205-230, 2003.

SANTANA, A.C. de. Análise do desempenho competitivo das agroindústrias de frutas do Estado do Pará. In: CONGRESSO BRASILEIRO DE ECONOMIA E SOCIOLOGIA RURAL, 2006, Fortaleza - CE. Brasília - DF: SOBER, 2006. v. 44, p. 1-20.

SANTANA, A.C. de. Elementos de economia, agronegócio e desenvolvimento local. Belém: GTZ; TUD; UFRA, 2005. p.133-142. (Série Acadêmica, 01).

SANTOS, Marcos Antônio Souza. Organização e competitividade das micro e pequenas empresas de artefatos de madeira do Estado do Pará. 2002. 158 f. Dissertação (Mestrado em Economia) Universidade da Amazônia. 
Zilda Joaquina Cohen Gama, Antônio Cordeiro de Santana,

Fernando Antônio Teixeira Mendes \& Ahmad Saeed Khan

SEBRAE. Metodologia de desenvolvimento de arranjos produtivos locais: Projeto Promos/Sebrae/Bid - versão 2.0. Disponível em: <www.sebrae.org.br>. Acesso em: 18 jan. 2005.

SHY, O. Industrial organization: theory and applications. London: MIT Press, 1997.

SILVA, M.C.N. Competitividade das agroindústrias de polpa de frutas das mesorregiões metropolitana de Belém e nordeste paraense (1996 a 2003). 2006. 130 f. Dissertação (Mestrado em Economia) - Universidade da Amazônia.

SPSS. SPSS Base 7.5 applications guide. Chicago: SPSS Inc., 1997.

TIROLE, J. The theory of industrial organization. Cambridge: MIT Press, 2001.

Abstract - The furniture industry of the Metropolitan Region of Belém provides many jobs opportunities and help to reduce the environmental impacts of sawmill, given that the shavings and wood residues of these firms are used as raw material. Factorial analysis was used to estimate the index of competitive performance (ICP) and to determine the competitive position of the firms in the furniture industry. The results showed that most of the furniture firms were located in an intermediate competitive level, which is due to the limiting factors such as lack of workforce, low technological level, difficult access to credit and low level of horizontal and vertical integration in the supply chain. The final results showed that only one, among the furniture firms, achieved a high competitive performance position.

Key words: Index of competitive performance (ICP), Factorial analysis, Furniture firms, Technology, State of Pará. 
REVISTA DE ECONOMIA E AGRONEGÓCIO, VOL.5, $N^{\circ} 1$ 